INPLASY

PROTOCOL

To cite: Yin et al. The diagnostic value of Diffusion tensor imaging (DTI) in Parkinson's disease: a metaanalysis. Inplasy protocol 202070098. doi:

10.37766/inplasy2020.7.0098

Received: 21 July 2020

Published: 21 July 2020

Corresponding author:

Xiaoqin Yin

1396501315@qq.com

Author Affiliation:

Affiliated Hospital of Chengdu

University of Traditional

Chinese Medicine

Support: None.

Review Stage at time of this submission: Preliminary searches.

Conflicts of interest: None.

\section{The diagnostic value of Diffusion tensor imaging (DTI) in Parkinson's disease: a meta-analysis}

Yin, XQ1; Liu, MJ2; Liao, Y3; Li, QZ4; Hou, XL5; Yang, DD6; Chu, X7; Zhu, C8; Jin, SG9.

Review question / Objective: A number of studies have shown that the PA, MD and ADC values of dopamine neurons lost in PD patients are different. Therefore, this paper discussed the diagnostic role of DTI in Parkinson's disease through systematic analysis.

Condition being studied: Parkinson's disease (PD) is an agerelated neurodegenerative disease of unknown etiology, second only to Alzheimer's disease in prevalence, The severe selective loss of neuronal pigmentation in SN is related to the formation of Lewy bodies and neuritis in specific areas of the brain. Neuroimaging can help us better understand the pathophysiology and symptoms of Parkinson's disease (PD) . Diffusion tensor imaging (DTI) is a non-invasive neuroimaging technology based on MRI, which can analyze the diffusion rate in brain tissue and help us better understand the pathophysiological changes of the disease.

INPLASY registration number: This protocol was registered with the International Platform of Registered Systematic Review and Meta-Analysis Protocols (INPLASY) on 21 July 2020 and was last updated on 21 July 2020 (registration number INPLASY202070098).

\section{INTRODUCTION}

Review question / Objective: A number of studies have shown that the PA, MD and ADC values of dopamine neurons lost in PD patients are different. Therefore, this paper discussed the diagnostic role of DTI in Parkinson's disease through systematic analysis.
Condition being studied: Parkinson's disease (PD) is an age-related neurodegenerative disease of unknown etiology, second only to Alzheimer's disease in prevalence, The severe selective loss of neuronal pigmentation in $\mathrm{SN}$ is related to the formation of Lewy bodies and neuritis in specific areas of the 
brain. Neuroimaging can help us better understand the pathophysiology and symptoms of Parkinson's disease (PD) . Diffusion tensor imaging (DTI) is a noninvasive neuroimaging technology based on MRI, which can analyze the diffusion rate in brain tissue and help us better understand the pathophysiological changes of the disease.

\section{METHODS}

Participant or population: Inclusion criteria: 1. Diagnosis of Parkinson's disease 2. The study is an observational study; 3 . The study should use DTI technology to screen PD patients and healthy controls; 4 . The study should have detailed information about FA and MD or ADC Data (MD and ADC are not completely necessary, but both indicators are descriptions of the total spread and should be included in the analysis). Reviews, editorials and case reports are not included.

Intervention: Not applicable.

Comparator: Healthy people.

Study designs to be included: Observational study.

Eligibility criteria: Inclusion criteria: 1. Diagnosis of Parkinson's disease 2. The study is an observational study; 3 . The study should use DTI technology to screen PD patients and healthy controls; 4 . The study should have detailed information about FA and MD or ADC Data (MD and ADC are not completely necessary, but both indicators are descriptions of the total spread and should be included in the analysis).

Information sources: We will primarily search Cochrane, PubMed, EMBASE, studies regardless of publication date, but only english will be included.for the missing information we will contact the author.Additionally, the bibliographies of relevant articles were scanned for further suitable literature.

Main outcome(s): Fractional anisotrop (FA).
Additional outcome(s): "Apparent diffusion coefficient, ADC", "mean diffusion coefficient (MD).

Quality assessment / Risk of bias analysis: Two authors will assess the risk of bias with the Cochrane Collaboration's tool for risk of bias assessment for all included studies.

Strategy of data synthesis: Continuous outcome variables were measured using mean differences (MDs) and corresponding $95 \%$ confidence intervals (CIs). Heterogeneity between studies was detected by Cochran's Q-test with $P<0.05$ as a significance level, and quantitatively measured through $\mathrm{I}^{2}$ statistic.A fixed effect model (FEM) was applied to perform the meta-analysis if the P-value of Cochran Qtests was more than 0.05 , otherwise, random effects model was utilized.

Subgroup analysis: Subgroup analysis based on study designs, otherwise Subgroup analyses will be performed (if data can be pooled) to assess neurodegenerative changes in different brain regions.

Sensibility analysis: We will conduct sensitivity analysis from the aspects of sample size, methodological quality and missing data, et al.

Country(ies) involved: China.

Keywords: Diffusion tensor imaging; Parkinson's disease; Meta-analysis.

Contributions of each author:

Author 1 - Xiaoqin Yin.

Author 2 - Meijun Liu.

Author 3 - Yang Liao.

Author 4 - Qizheng Li.

Author 5 - Xiaolin Hou.

Author 6 - Dongdong Yang.

Author 7 - Xin Chu.

Author 8 - Chan Zhu.

Author 9 - Shuoguo Jin. 